\title{
EFFECTIVE USE OF METALLIC Z-PINS FOR COMPOSITES' THROUGH- THICKNESS REINFORCEMENT
}

Beene M'membe*, Mehdi Yasaee ${ }^{1}$, Stephen R. Hallett, Ivana K Partridge

Bristol Composites Institute (ACCIS), University of Bristol, Bristol, BS8 1TR, UK

\section{*Beene.MMembe@bristol.ac.uk}

\begin{abstract}
Z-pins offer effective through-thickness reinforcement for laminated composites. Various studies have however shown that metal Z-pins are less effective at bridging Mode I delaminations than carbon-fibre composite Z-pins, due to poor interfacial bonding with the laminate. This is exacerbated by high thermal mismatch between the metallic Z-pins and the laminate. This study investigates inserting metallic Z-pins at angles offset from the laminate normal, to improve the Mode I bridging in composites. The effects on the apparent fracture toughness under pure and mixed Mode I/II loads using single pin specimens is investigated. Results show that, unlike orthogonally inserted metal Z-pins, inclined Z-pins exhibit high energy absorption throughout the mixed mode range. Double Cantilever Beam (DCB) tests show that the inclined metal Z-pins increase the Mode I apparent fracture toughness by a factor of 2 compared to traditional carbon fibre Z-pins. In End Loaded Split (ELS) tests, the Mode II apparent fracture toughness of inclined stainless steel Z-pins, although less than their uninclined
\end{abstract}

\footnotetext{
${ }^{1}$ Cranfield University, Cranfield MK43 0AL, United Kingdom (present address)
} 
equivalent, is greater than that of carbon fibre Z-pins.

Keywords: Fracture Toughness, Inclined Insertion, Metal Z-pins

\section{Introduction}

Z-pins are small rods inserted in the through the thickness of a laminate. They are used to improve the through-thickness properties of laminated composites. Z-pins most commonly take the form of thin pre-cured carbon fibre rods, inserted into an uncured pre-preg laminate. Metallic Z-pins are the most commonly used Z-pin type after composite Z-pins [1]-[6]. Metallic Z-pins differ from carbon-fibre through-thickness reinforcements because of their capacity to absorb large amounts of fracture energy via plastic deformation. In addition, metallic Z-pins can also possess additional properties, such as electromagnetism, which are potentially attractive for multifunctional laminates [7], [8]. However, the use of metal Z-pins is hindered by their high specific density, high thermal expansion coefficients, and depending on the electro-potential value of the metal, high probability of galvanic corrosion with the carbon fibres in the laminate [9].

Metal Z-pins tend to have poor interfacial properties with composite materials, therefore surface treatments are necessary to ensure high fracture toughness under Mode I loading [1]-[3]. In the literature, aggressive surface treatment techniques such as targeted forging on the Z-pin surface and aqua regia solutions have been investigated to increase the bond strength of metal Z-pins with the laminate [1], [2]. Organosilane-coupling agents have also been shown to be effective at improving Mode I delaminations through the formation of polar bonds [3]. The same study [3] also demonstrated the importance of selecting Z-pin materials that can withstand the pull-out process, and more critically 
it highlighted that the use of surface treatments on metallic Z-pins, if poorly tailored with the laminate, may lead to low energy failure mechanisms.

Previous studies have shown that the use of inclined Z-pins is an effective means of increasing energy absorption of Z-pinned composites [4], [10], [11]. The benefits of inclined metallic reinforcements, particularly for pull-out applications, are well known in civil engineering applications where stainless steel rods are inserted in cement [12]. One of the earliest works on inclined metallic reinforcements was conducted at University of Oxford in 1974 [13]. A key finding from this study was that the benefit of increasing the peak load, and consequently the energy absorption of inclined metallic reinforcements loaded in Mode I, culminates at $45^{\circ}$ [13]. The effects of high insertion angles of metal Z-pins under shear dominated loads are not well established beyond the study carried out by Cartie et. al. [4]. In general, Z-pins loaded with the nap failed via pull-out while those loaded against the nap fail via rupture for insertion angles greater than $15^{\circ}$. It is possible that the rupture failure observed could be a result of strong interfacial properties of titanium Z-pins with the laminate (average frictional stress was $80 \%$ of the T300/BMI Z-pins). Cartie et. al. [4] did not establish a clear pattern with respect to energy absorption under Mode I/II loads from their data set.

This study describes how the energy absorption of metallic Z-pins can be significantly increased without using any surface treatments. The Z-pins are inserted in composite laminates and tested under mixed-Mode I/II loads. The effect of insertion angle on the bridging performance is characterised on a sub-coupon level using single pin specimens and at coupon level using DCB and ELS specimens. Finally, key highlights and 
recommendations are made, thereby completing the knowledge gap on the mixed mode response of inclined metallic Z-pins.

\section{Materials and methods}

The single pin specimens were manufactured from a quasi-isotropic laminate with a $[0,+45,90,-45]_{4 \mathrm{~s}}$ layup in the top half and a $[90,-45,0,+45]_{4 \mathrm{~s}}$ layup in the bottom half. The laminate was made from carbon/epoxy IM7/8552 pre-preg tape, supplied by Hexcel. A layer of PTFE release film was inserted at the mid-plane of the laminate to create an artificial delamination. The specimens are designed to have a $90^{\circ}$ ply angle mismatch at the mid-plane to avoid any nesting of the plies. The test specimen dimensions were $20 \times 20 \times 8 \mathrm{~mm}$ (see Figure 1).
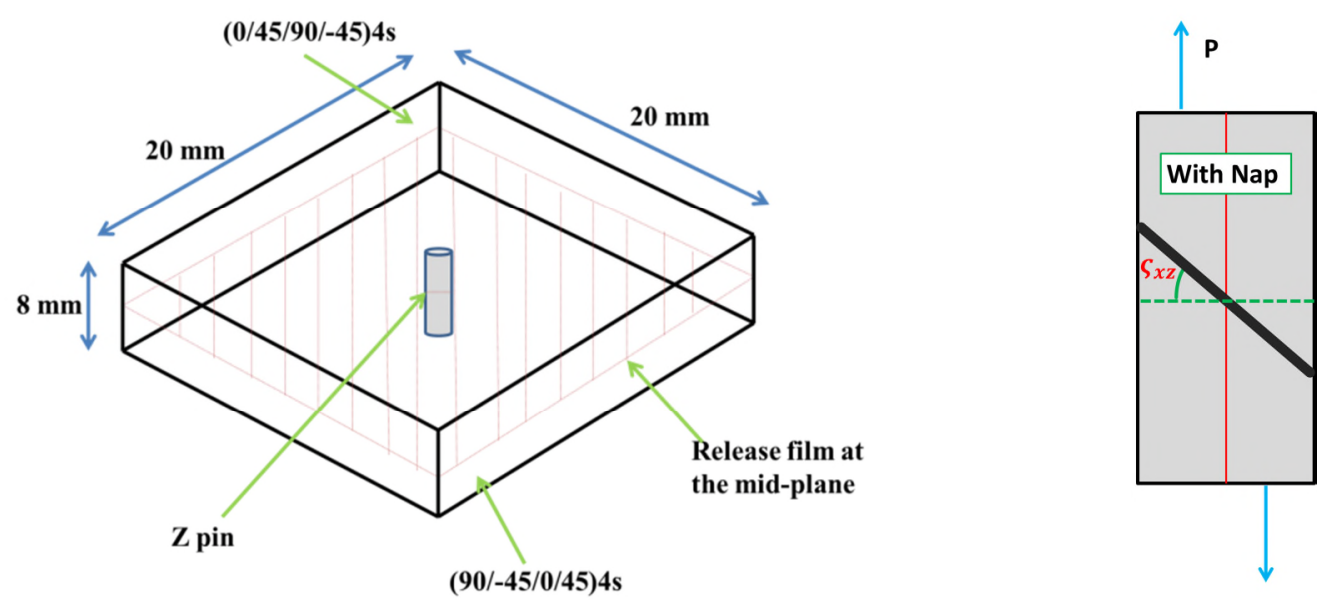

Figure 1 Schematic diagram of single Z-pin specimens

The specimens were reinforced with $0.3 \mathrm{~mm}$ diameter stainless steel Z-pins, supplied by Classic Plus, inserted at $0^{\circ}$ and $45^{\circ}$. The variation of the insertion angles is shown in

Table 1. All tests were carried out with the specimens loaded with the nap, using an 
Instron 8872 universal testing machine with a $1 \mathrm{kN}$ load cell at a rate of $0.5 \mathrm{~mm} / \mathrm{min}$ until failure (see Figure 1 ). Further details on the test procedure can be found in Yasaee et al. [14] and M'membe et al. [10].

Table 1. Effective insertion angles $(\boldsymbol{\varsigma})$, based on process commissioning data, standard deviation in brackets

\begin{tabular}{ll}
\hline Intended Insertion Angle $\left(\boldsymbol{S}_{\boldsymbol{x} z}{ }^{\circ}\right)$ & Effective Insertion Angle $\left(\boldsymbol{\zeta}^{\circ}\right)$ \\
\hline 0 & $1.62(1.22)$ \\
45 & $55.6(8.01)$ \\
\hline
\end{tabular}

Figure 2 shows the geometry of the DCB specimens. Z-pins in these specimens were inserted at an areal density of $0.5 \%$. For inclined pins, an insertion angle of $45^{\circ}$ was used, with inclination alternating between $+\theta$ and $-\theta$ for any given row running across the beam width, as shown on the inset in Figure 2. The tests were carried out in accordance with the ASTM D5528-01 standard for testing interlaminar fracture toughness of composites [15]. Each specimen was machined from a panel with a layup of $\left([0,-45,90,+45]_{4 \mathrm{~s}}\right)_{\mathrm{s}}$ to a size of $180 \times 20 \mathrm{~mm}$ with a nominal thickness of $8 \mathrm{~mm}$. The initial crack length $a_{o}$ was $50 \mathrm{~mm}$ with respect to the loading line. Piano hinges were glued on to the specimen surface using Redux 810 epoxy adhesive (supplied by Hexcel). The specimens were tested at a displacement rate of $2 \mathrm{~mm} / \mathrm{min}$ using an Instron 8872 universal machine with a calibrated $10 \mathrm{kN}$ load cell. Crack propagation was recorded using a high resolution camera capable of resolving the crack tip to a $\pm 0.5 \mathrm{~mm}$ accuracy. The fracture toughness of the specimens was determined using equation Error! Reference source not found.). The crack length $(a)$ used to calculate the 
fracture toughness $\left(\mathrm{G}_{\mathrm{I}}\right)$ was modified by a geometry independent additional parameter $\Delta_{\mathrm{I}}$, in order to account account for deflection and rotation at the crack tip [16].

$G_{I}=\frac{3 P}{2 b} \frac{\delta}{\left(a+\left|\Delta_{\mathrm{I}}\right|\right)} F$
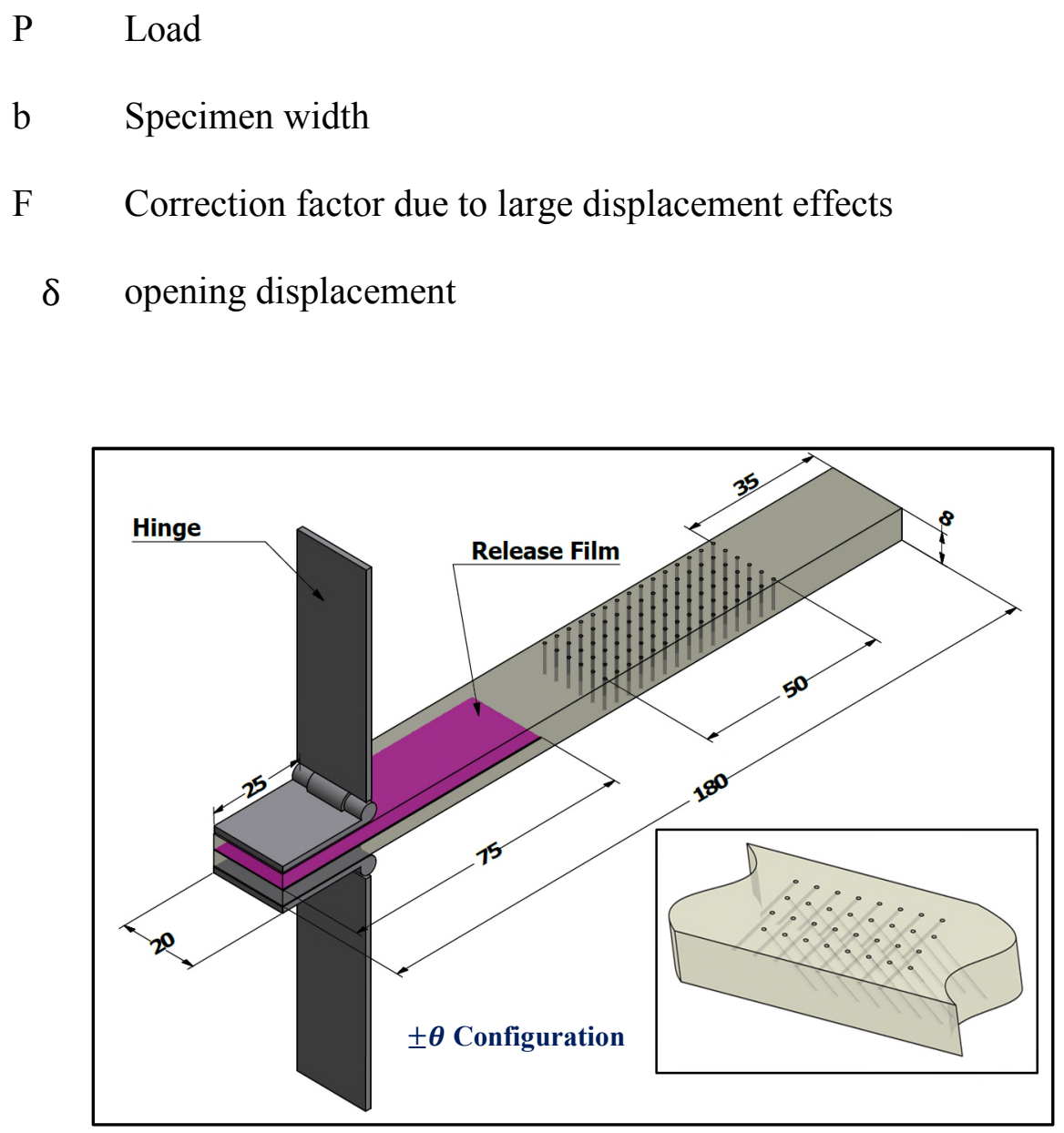

Figure 2 Schematic diagram of DCB specimens

In pure Mode I tests such as those carried out for single pin specimens, Z-pins are loaded with a purely axial force. However, in DCB specimens, the Z-pins near the crack front have axial forces and bending moments exerted on them [5]. For carbon fibre Z- 
pins normally reported in the literature, the contribution of bending moments can be ignored because they are negated by the growth of cracks along the Z-pin longitudinal axis [5]. However, bending effects cannot be ignored for the metallic Z-pins and must be considered in the DCB analysis [5]. Figure 3 shows a schematic diagram of the deformation of inclined and non-inclined Z-pins in DCB specimens. The opening displacements $\delta$ is given by

$\delta=\delta_{u}+\delta_{l}$

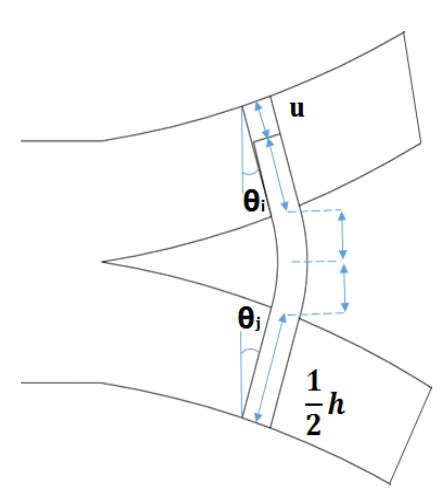

(a)

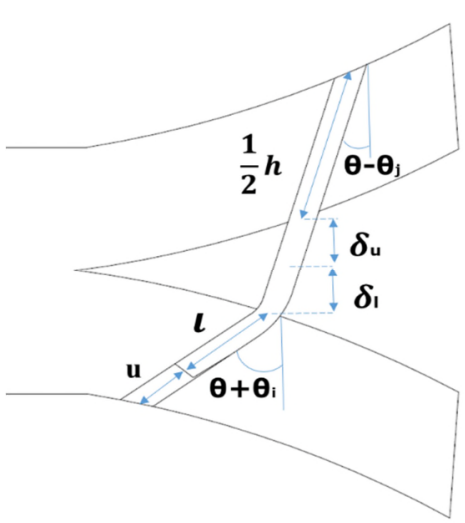

(b)

Figure 3 Deformation of a Z-pin in a DCB specimen for (a) uninclined and (b) inclined pins

Experimental observations have shown that pull out typically occurs from one half of the specimen. Therefore, with this assumption, the embedded length of the Z-pin from the non-pull-out arm of the DCB specimen is $\frac{1}{2} h$. There are cases where the other half also exhibits partial pull-out (usually less than $8 \%$ the full thickness). This has the effect of producing longer pull-out lengths and contributes to the scatter in Figure 6. This 
behaviour is enhanced in Z-pins with very low interfacial bond strength, such as the steel pins studied here. $l$ is the length of the Z-pin which is still embedded in the specimen from the pull-out arm. The rotation of the beam in the DCB specimens is $\theta_{i}$ and $\theta_{j}$ for the pull-out arm and the non-pull-out arm respectively. For an inclined Z-pin the effective rotation can be derived from the geometry of the Z-pin as shown in Figure 3(b) where $\theta$ is the effective insertion angle.

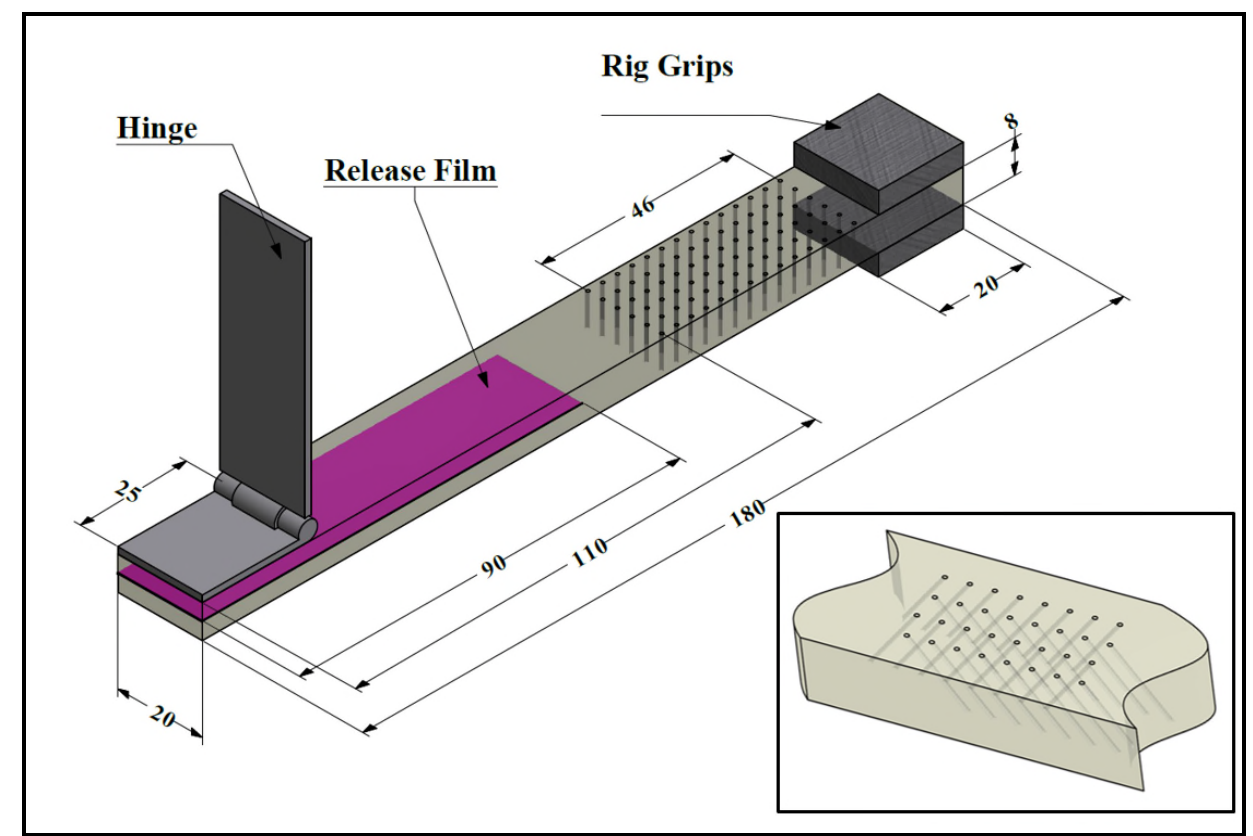

Figure 4 shows the specimen geometry and test set-up for the ELS test. The laminate lay-up and external dimensions are identical to the DCB specimens. The specimens were tested in accordance with the European Structural Integrity Society (ESIS-TC4 0104-02) test procedure at a load rate of $2 \mathrm{~mm} / \mathrm{min}$ [17]. 


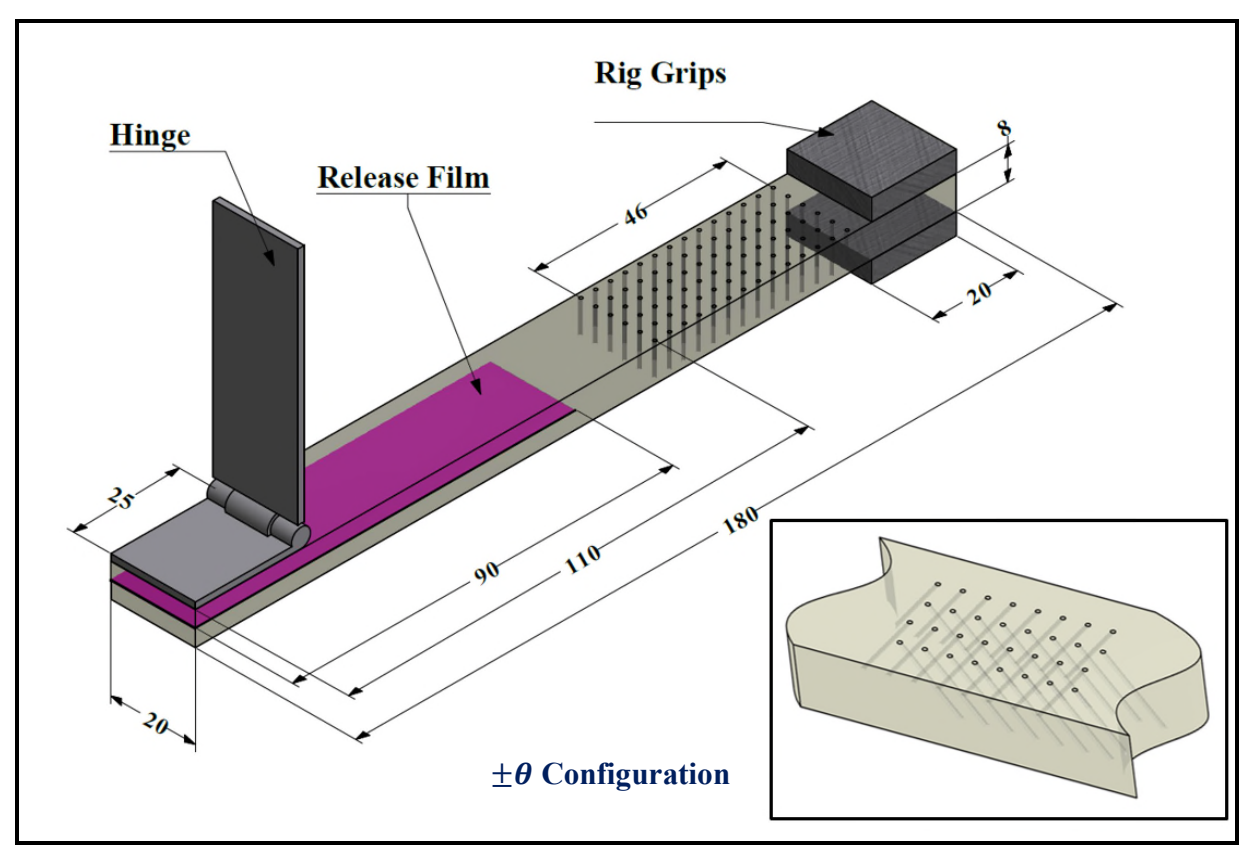

Figure 4 Schematic Diagram of ELS Specimens

The tests were carried out on a calibrated 8872 Instron machine with a $10 \mathrm{kN}$ load cell. The specimens were clamped $135 \mathrm{~mm}$ ahead of the load-line. Prior to testing, a natural crack was propagated $5 \mathrm{~mm}$ beyond the film insert edge using a Mode II load. The fracture toughness of specimens is calculated using equation (3). The crack displacement correction factor is equal to $0.42 \Delta_{\mathrm{I}}[18]$. The flexural modulus $\mathrm{E}_{\mathrm{f}}$ is 55.21 GPa for the material used in this study.

$$
\mathrm{G}_{\mathrm{IIC}}=\frac{9 \mathrm{P}^{2}\left(\mathrm{a}+\Delta_{\mathrm{II}}\right)^{2}}{4 \mathrm{~b}^{2} \mathrm{E}_{f}\left(\frac{\mathrm{h}}{2}\right)^{3}}\left[1-\theta_{1}\left(\frac{\delta}{\mathrm{L}}\right)^{2}-\theta_{2}\left(\frac{\delta \mathrm{I}_{1}}{\mathrm{~L}^{2}}\right)\right]
$$

L Distance from the load line to the clamped line

h Specimen thickness 
$\theta_{1}$ and $\theta_{2} \quad$ Correction factor parameters

The presence of large-scale bridging from Z-pins in DCB and ELS specimens means that these tests, which normally produce laminate material properties, have a structural component that depends on the Z-pin material properties, their layout in the specimens, as well as other parameters such insertion depth, specimen size, etc. As a result, the fracture toughness values derived from the tests in this section are termed 'apparent fracture toughness' since they do not determine material properties as defined by the test standards.

\section{Results and discussion}

\subsection{Bridging behaviour of inclined metallic Z-pins}

The Mode I/II load-displacement and energy absorption data for the single pin specimens are shown in Figure 5 and Figure 6. All the specimens tested failed via pullout at all the mixed Mode load angles tested in this study. In Mode I, the maximum load sustained during pull-out for the 0 degree stainless steel Z-pins is significantly lower than that of traditional carbon fibre Z-pins [14]. This is caused by poor interfacial properties between metallic Z-pins and the laminate, which results in a relatively low frictional pull-out coefficient. The poor interfacial bonding is exacerbated by thermal contractions of the Z-pins after curing in the autoclave, which lead to premature debonding of the Z-pins. The Mode I energy absorption of the inclined stainless steel Zpins is significantly increased compared to orthogonally inserted pins. The increase is a result of increased friction due to snubbing between the Z-pin and the laminate, as well as plastic deformation of the Z-pin at the exit point of the half laminate during pull-out 
(see Since the embedded length of the Z-pins increases with insertion angle, the energy absorption required for the pull-out of Z-pins inserted at $45^{\circ}$ should be increased by a factor of 1.41 over that of $0^{\circ}$ inserted Z-pins, if one assumes the friction conditions were constant between the two cases. Figure 8, which shows the pull-out force of the Z-pins normalised by pin length, indicates that the increase in Mode I loads for the inclined pins is far above that anticipated by just the increased length. The energy absorption of the inclined metal Z-pins in Mode I, on average, is almost twice that of carbon fibre Zpins tested in the same laminate under the same loading environment [10], [20]. Thus, the results here show that an inclined ductile metal Z-pin has more effective energy absorption mechanisms, via plastic deformation and increased frictional pull-out due to snubbing compared to a non-inclined carbon fibre Z-pin which absorbs energy via frictional pull-out alone. Inclined metal Z-pins also exhibit high apparent fracture energy in Mode II compared to carbon fibre Z-pins [10], [20]. Thus, by using inclined metallic Z-pins, it is possible to have a metal Z-pin that provides high energy absorption across the whole range of Mixed Mode I/II angles without the use of surface treatments.
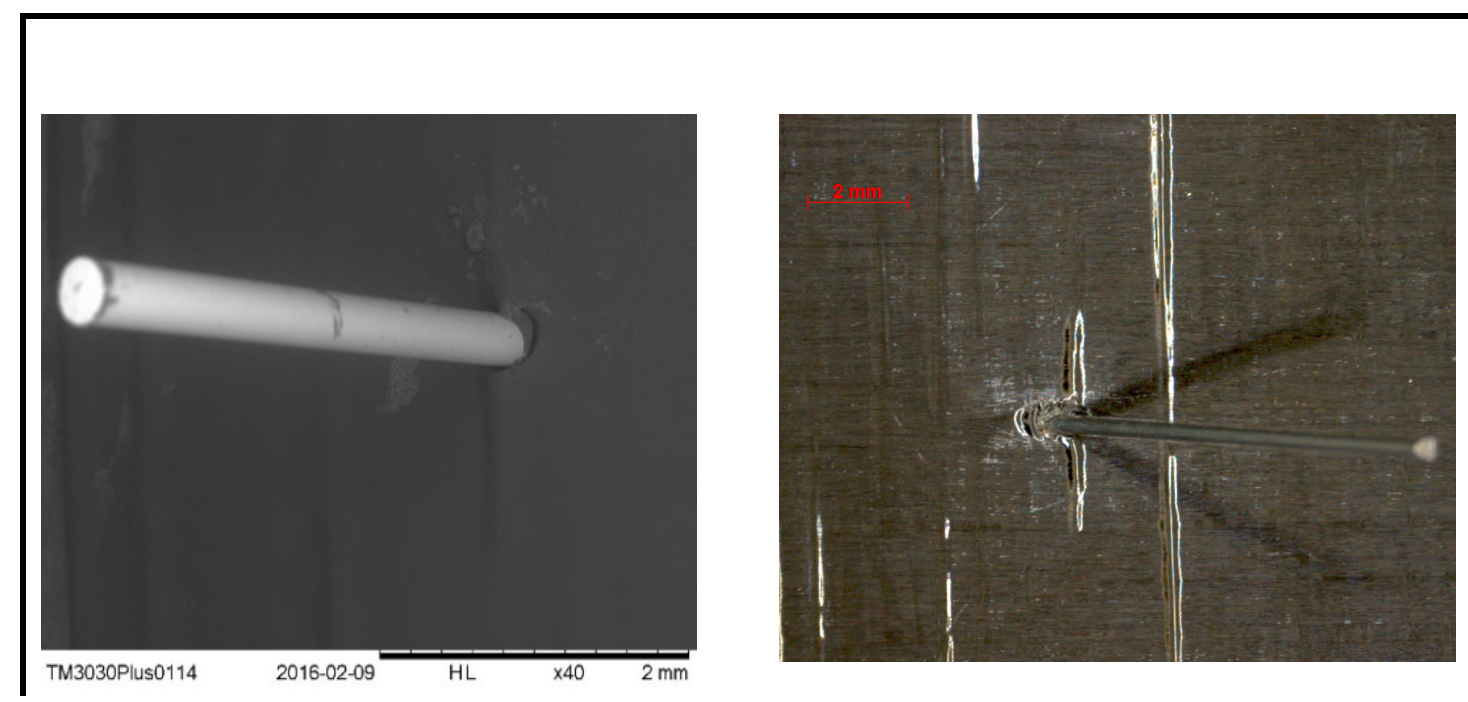

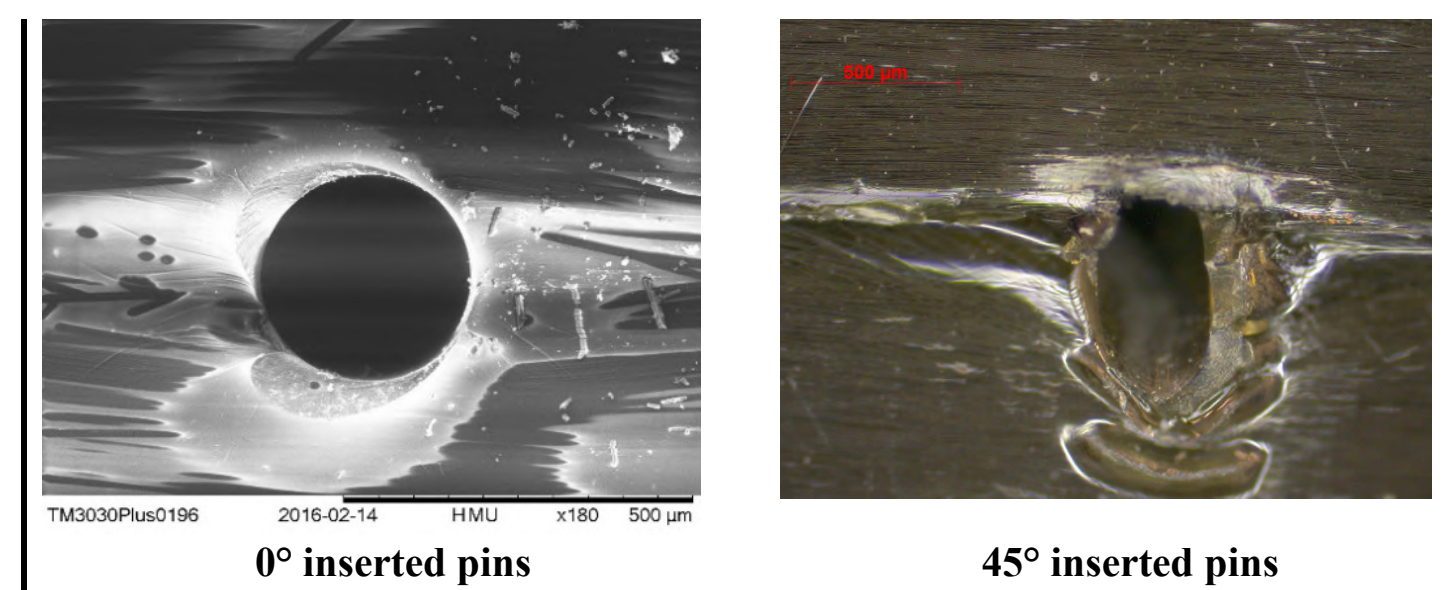

$45^{\circ}$ inserted pins

Figure 7). The load drop seen on the load-displacement plot is a result of matrix spalling due to high stress concentrations at the exit point [12].

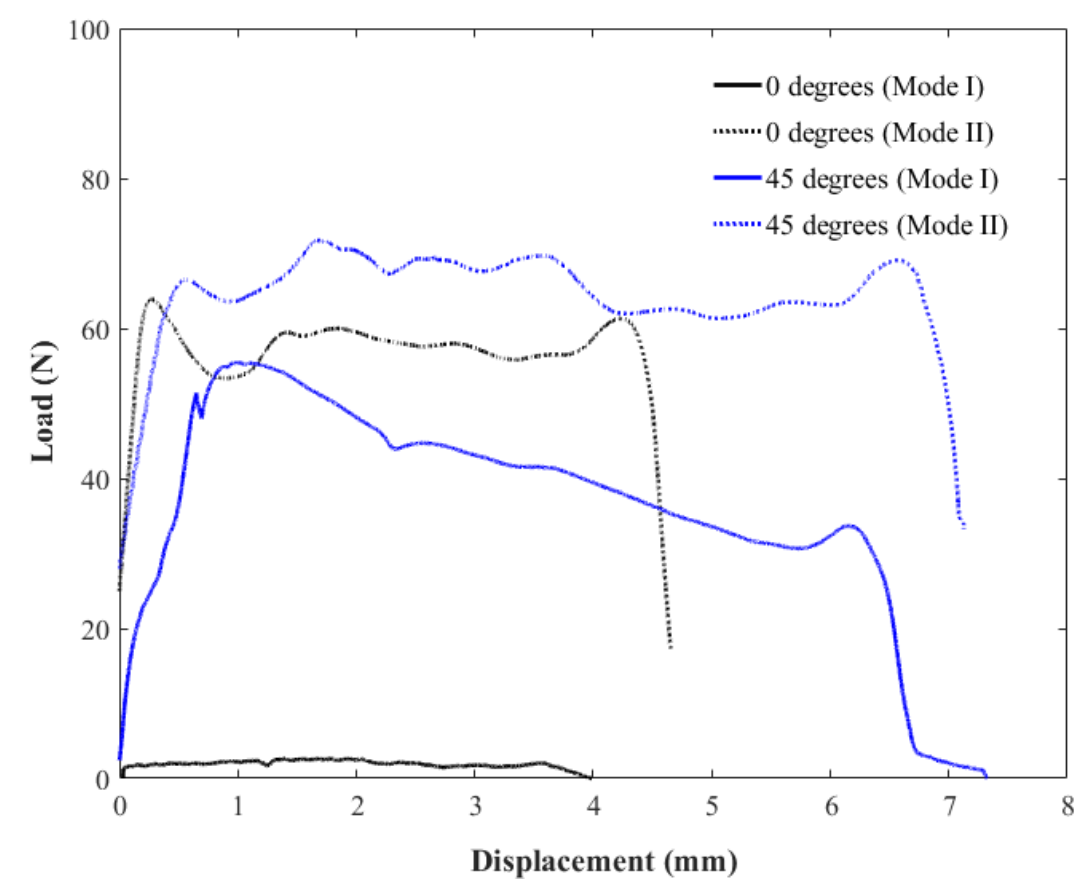

Figure 5. Typical load-displacement curves for non-inclined and inclined stainless steel Z-pins. (Single pin tests) 


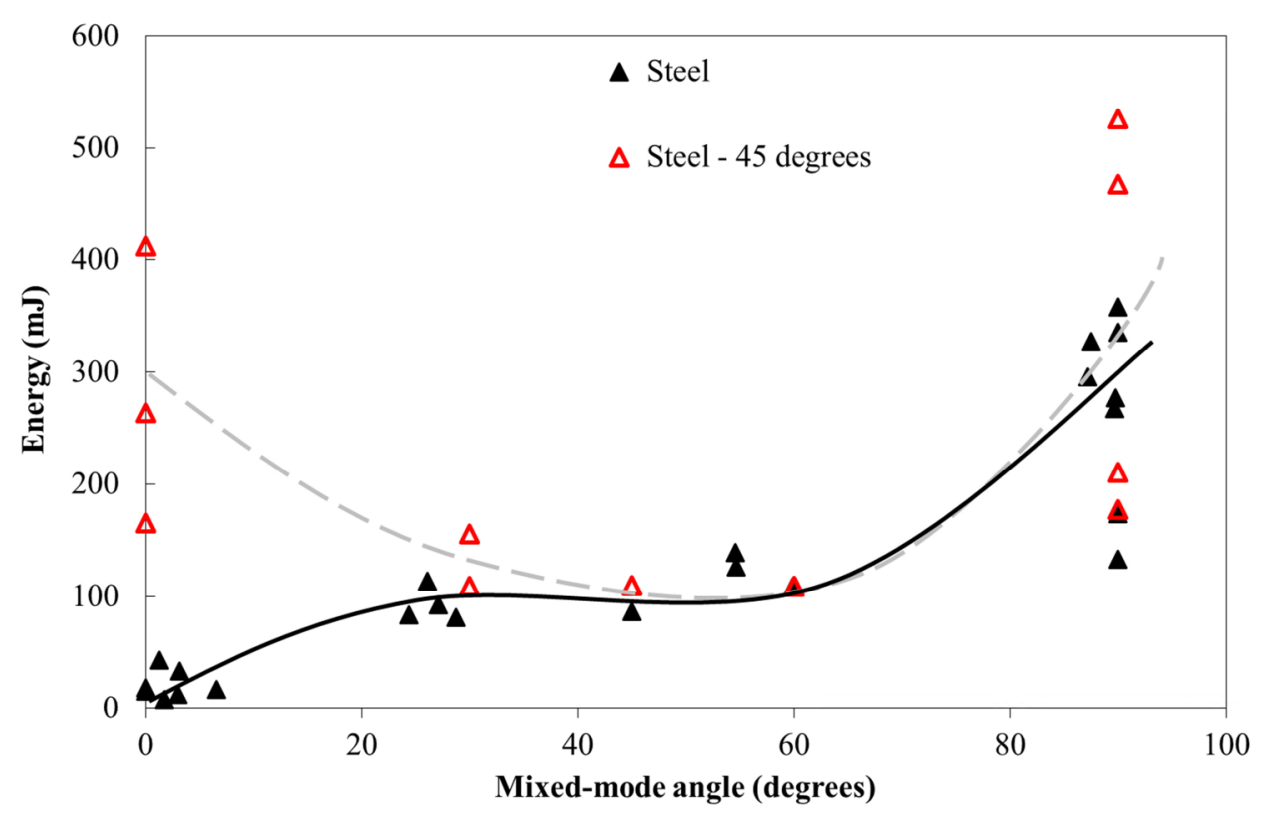

Figure 6. Energy absorption for inclined stainless steel Z-pins compared non-inclined Zpins. The lines drawn are to illustrate the general trend in the data and are not fitted curves. (Single pin tests)

Since the embedded length of the Z-pins increases with insertion angle, the energy absorption required for the pull-out of Z-pins inserted at $45^{\circ}$ should be increased by a factor of 1.41 over that of $0^{\circ}$ inserted Z-pins, if one assumes the friction conditions were constant between the two cases. Figure 8, which shows the pull-out force of the Z-pins normalised by pin length, indicates that the increase in Mode I loads for the inclined pins is far above that anticipated by just the increased length. The energy absorption of the inclined metal Z-pins in Mode I, on average, is almost twice that of carbon fibre Zpins tested in the same laminate under the same loading environment [10], [20]. Thus, the results here show that an inclined ductile metal Z-pin has more effective energy absorption mechanisms, via plastic deformation and increased frictional pull-out due to snubbing compared to a non-inclined carbon fibre Z-pin which absorbs energy via 
frictional pull-out alone. Inclined metal Z-pins also exhibit high apparent fracture energy in Mode II compared to carbon fibre Z-pins [10], [20]. Thus, by using inclined metallic Z-pins, it is possible to have a metal Z-pin that provides high energy absorption across the whole range of Mixed Mode I/II angles without the use of surface treatments.

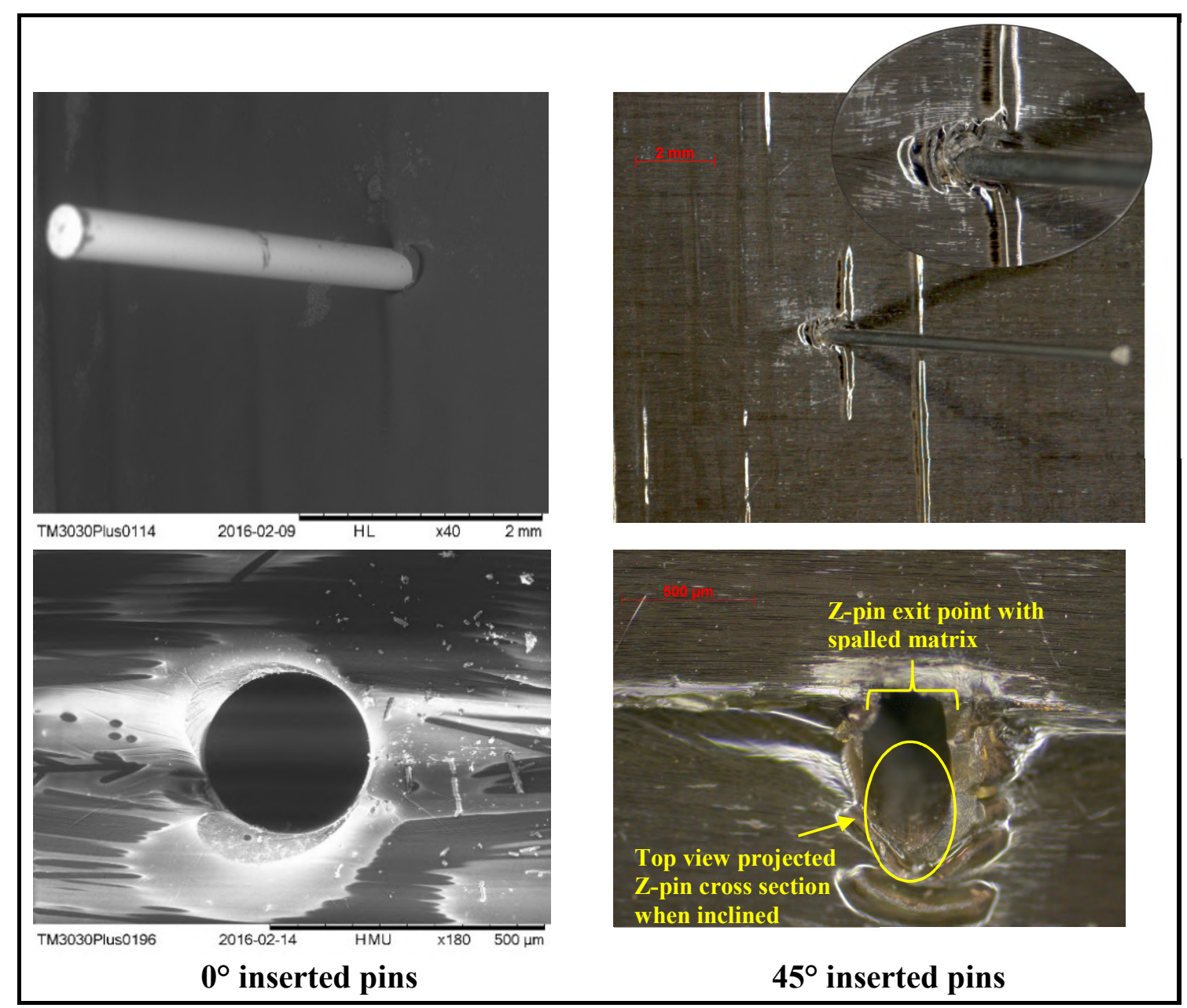

Figure 7. Micrograph of the fracture surface for stainless steel Z-pins tested in Mode I 


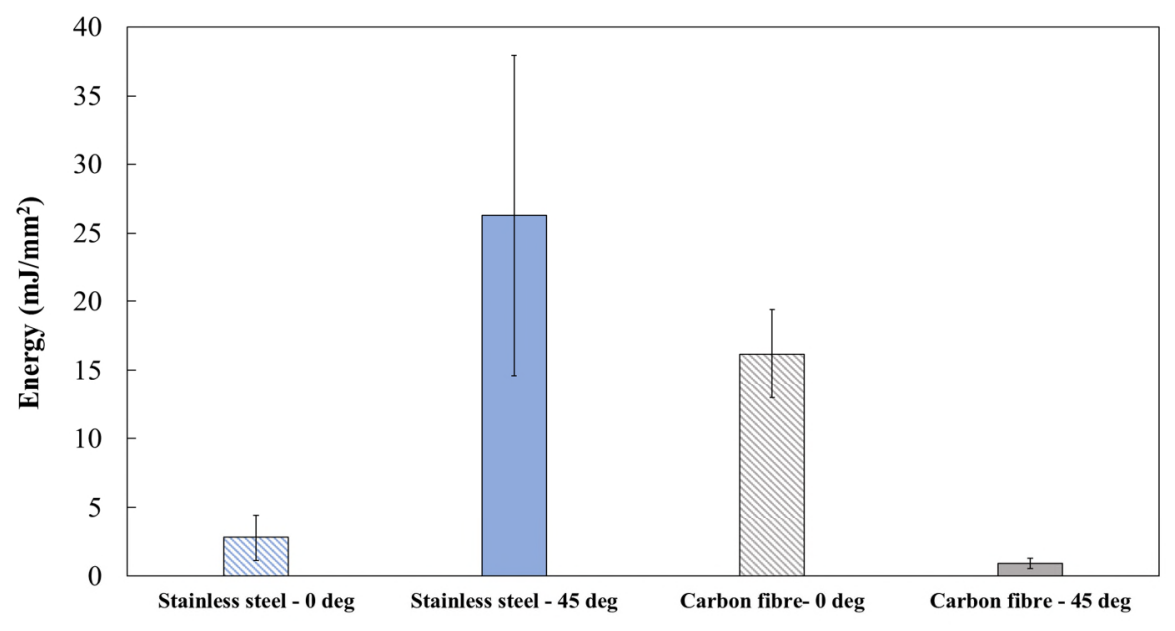

Figure 8. Comparison of Mode I average energy absorption of stainless Z-pins normalized by unit length, carbon-fibre data from [10], [18]. (Single pin tests)

\subsection{Mode I fracture toughness}

The Mode I R-curve plots in

Average $G_{I} \underline{\left(J / m^{2}\right)}$

\begin{tabular}{l|lll}
\hline Material & Unpinned & Carbon fibre & $\pm \theta$ Stainless steel \\
Initiation (CoV\%) & $234(12.0)$ & $225(24.8)$ & $260(2.40)$ \\
Propagation (CoV\%) & $283(11.9)$ & $7848(5.33)$ & $24168(9.74)$ \\
\hline
\end{tabular}

Figure 9 show the effect of different Z-pin materials and configurations on apparent fracture toughness of DCB Z-pinned specimens. The apparent fracture toughness shown on these plots is averaged across 4 specimens for each type Z-pin material, at regular crack lengths. As shown in

\section{Average $G_{I} \underline{\left(J / m^{2}\right)}$}

\begin{tabular}{l|ll}
\hline Material & Unpinned $\quad$ Carbon fibre $\quad \pm \theta$ Stainless steel
\end{tabular}


Initiation (CoV\%)

Propagation (CoV\%)
$234(12.0)$

$225(24.8)$

$283(11.9)$

Figure 9, the fracture toughness in Mode $\mathrm{I}\left(\mathrm{G}_{\mathrm{I}}\right)$ is unaffected by the presence of Z-pins before the crack tip reaches the Z-pinned region. As the crack propagates through the Zpinned region, the apparent $\mathrm{G}_{\mathrm{I}}$ rises steeply until steady state propagation is achieved (i.e. when the number of active Z-pins in the crack bridging zone remains constant as the crack propagates through the remaining Z-pinned area). For inclined stainless steel Z-pinned specimens, the apparent fracture energy needed for continued crack propagation under steady state conditions could not be sustained by the specimen arms. Consequently, the specimen arm failed through bending before the crack had propagated through the Z-pinned region. The apparent propagation fracture toughness shown here, by the inclined metal pins $\left(\sim 24000 \mathrm{~J} / \mathrm{m}^{2}\right)$, is the highest ever reported to date in the literature for Z-pinned specimens of equivalent areal density. It is also worth noting that the inclined Z-pins had higher fracture than the carbon fibre Z-pins even at low crack lengths (see Figure 10). 

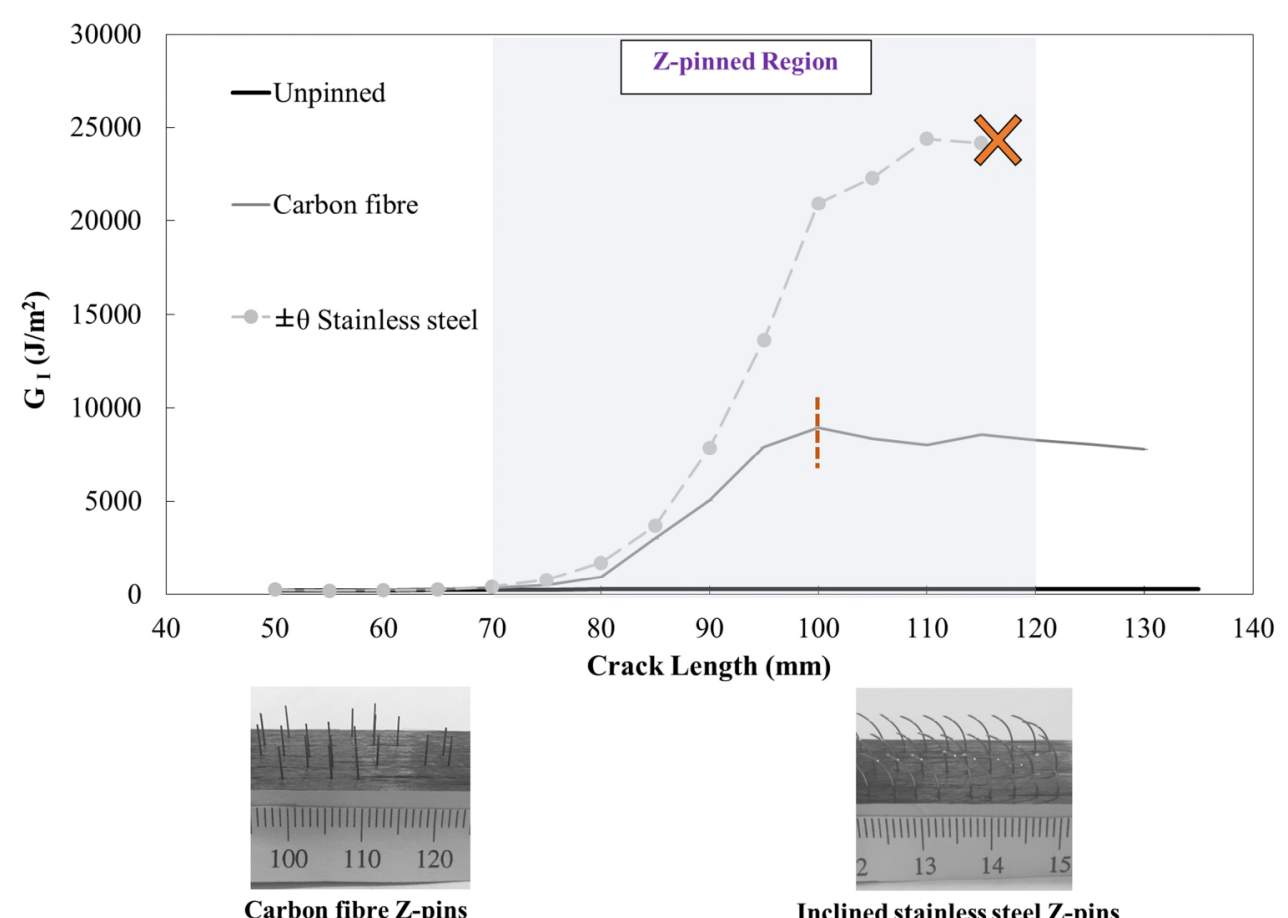

\section{Average $G_{I}\left(\mathrm{~J} / \mathrm{m}^{2}\right)$}

\begin{tabular}{l|lll}
\hline Material & Unpinned & Carbon fibre & $\pm \theta$ Stainless steel \\
Initiation (CoV\%) & $234(12.0)$ & $225(24.8)$ & $260(2.40)$ \\
Propagation (CoV\%) & $283(11.9)$ & $7848(5.33)$ & $24168(9.74)$ \\
\hline
\end{tabular}

Figure 9 Averaged R-curves for unpinned and Z-pinned specimens ( $0.5 \%$ areal density).

The vertical, dashed lines show the beginning of a fully developed bridging zone length.

The ' $X$ ' on the inclined stainless steel Z-pins represents arm failure of the specimens during testing. (DCB test) 


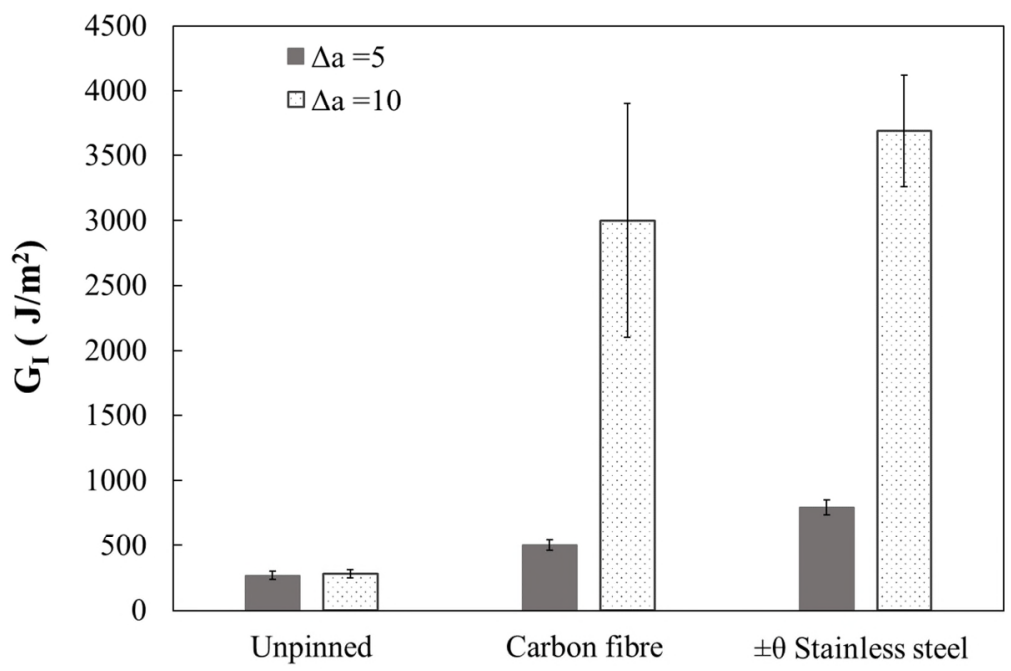

Figure 10 Average apparent $\mathrm{G}_{\mathrm{I}}$ at $\Delta \mathrm{a}=5 \mathrm{~mm}$ and $\Delta \mathrm{a}=10 \mathrm{~mm}$. (Mode I DCB test)

The bridging zone length of the Z-pins can be estimated by calculating the crack length (a) from the beginning of the Z-pinned region until a steady state $\mathrm{G}_{\mathrm{c}}$ value at propagation is achieved. It can be seen from

Average $G_{I}\left(J / m^{2}\right)$

\begin{tabular}{l|lll}
\hline Material & Unpinned & Carbon fibre & $\pm \theta$ Stainless steel \\
Initiation (CoV\%) & $234(12.0)$ & $225(24.8)$ & $260(2.40)$ \\
Propagation (CoV\%) & $283(11.9)$ & $7848(5.33)$ & $24168(9.74)$ \\
\hline
\end{tabular}

Figure 9 that the inclined stainless steel Z-pins have a longer bridging zone length than the carbon fibre Z-pins. This was caused by the increase in embedded length of inclined Z-pins, which were on average $37.5 \%$ longer than orthogonally inserted Z-pins. A similar effect has been observed in DCB specimens of stitched composites with an insertion angle of $45^{\circ}$, where the bridging zone length was increased by $37 \%$ with respect to the uninclined stitches [21]. The exact effect of Z-pin insertion length on the 
increase in bridging zone length cannot be determined from this study due to the unconventional failure mode of the inclined stainless steel specimens.

\subsection{Mode II fracture toughness}

Previous studies [10] have shown that inclining composite Z-pins improves the $\mathrm{G}_{\mathrm{II}}$ values compared to the conventionally inserted $0^{\circ} \mathrm{Z}$-pins when tested on ELS specimens. This study expands on the previous research by analysing the effect of material properties on inclined Z-pins using a $\pm 45^{\circ}$ configuration (see Figure 11 ). The results show that the effective Z-pinned region, that is the length along the longitudinal axis of the specimen where all Z-pins (with the nap and against the nap) can fully contribute to delamination resistance across the cross section of the specimen, is significantly reduced for inclined Z-pins. However, the length of specimen where Zpins in general are contributing to delamination resistance is longer. Therefore, inclined specimens at the initiation crack length $(\mathrm{a}=80 \mathrm{~mm})$ have active Z-pins loaded against the nap which increases the average $G_{I I}$ initiation values. Similarly, the apparent $G_{I I}$ for crack lengths greater than $120 \mathrm{~mm}$ only represents Z-pins loaded with the nap.

Although ELS tests produce a pure Mode II crack tip, the development of small crack openings between the beam halves as they shear against each other means that Z-pins in the crack wake are subjected to mixed loadings up to 0.6 as a ratio of Mode I to II [22]. For the specimens tested at $0.5 \%$ areal density, a fully developed bridging zone has not been achieved, therefore comparisons between the various Z-pins are made at crack lengths in the development stage of the bridging zone length. 
As expected from the two materials tested, stainless steel Z-pins have the higher apparent fracture toughness in the range tested. The fracture toughness of carbon fibre Z-pins is reduced due to their tendency to fail via rupture at small sliding displacements. Since stainless steel Z-pins are more ductile, they contribute bridging tractions at much higher sliding displacements compared to brittle carbon fibre pins which tend to fracture at high sliding displacements. This also inevitably means than the Mode II bridging zone lengths of the stainless steel Z-pins (which have not been reached in is study) are longer than that of carbon fibre Z-pins.

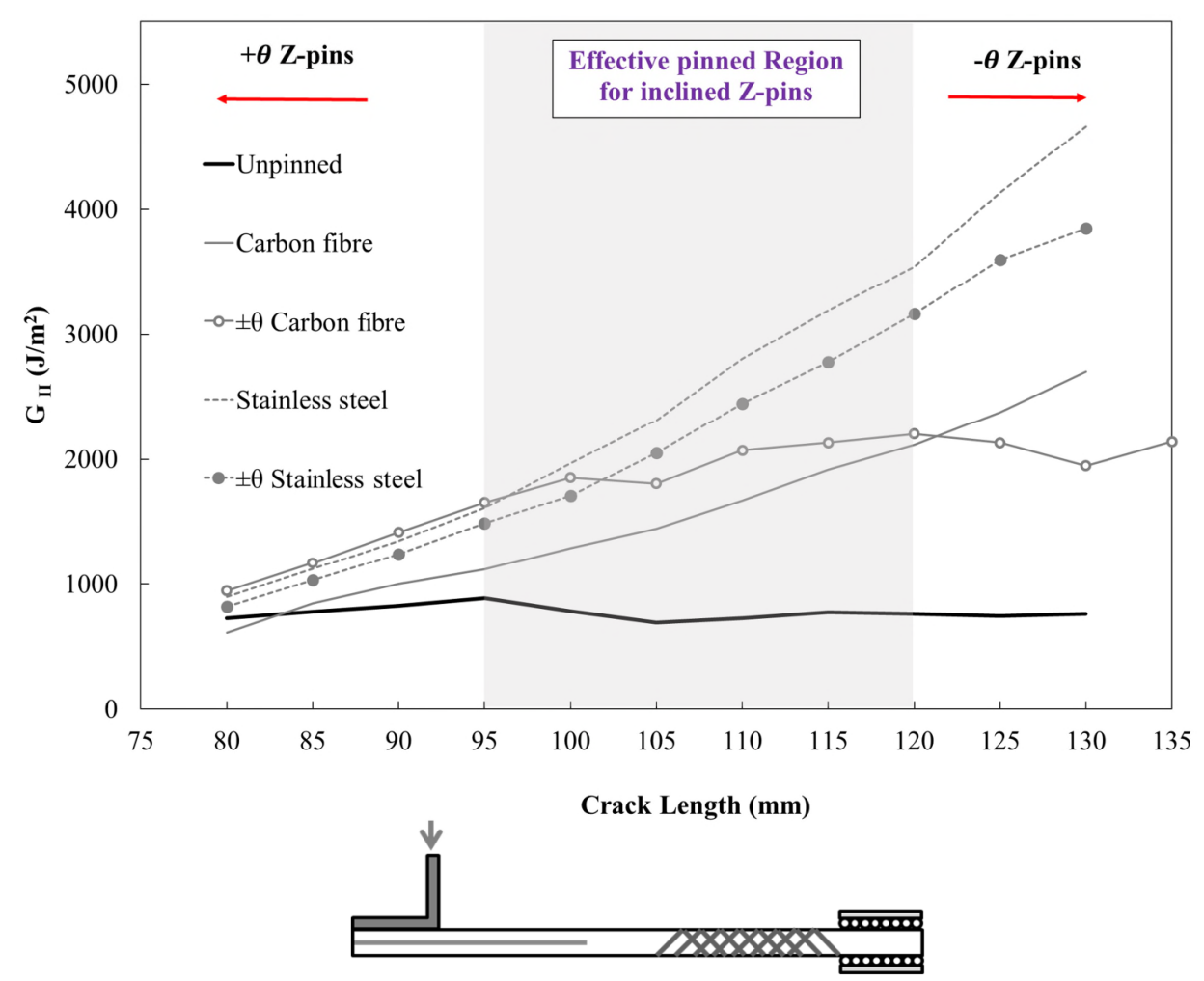

\section{$\underline{G}_{\underline{I I}}$ at Initiation $\left(\mathrm{J} / \mathrm{m}^{2}\right)$}

\begin{tabular}{l|lllll}
\hline Material & Unpinned & Carbon & Stainless & $\pm \theta$ Carbon & $\pm \theta$ Stainless \\
& & fibre & steel & fibre & steel \\
Initiation & 758 & 616 & 679 & 991 & 819
\end{tabular}




\begin{tabular}{l|lllll}
$\operatorname{CoV}(\%)$ & 11.3 & 21.9 & 22.4 & 9.6 & 11.6
\end{tabular}

Figure 11. Averaged R-curves for inclined Z-pins at $0.5 \%$ areal density. The effective Z-pinned region has been calculated based on an average insertion angle. (ELS test) In a $\pm \theta$ configuration for ELS specimens, $50 \%$ of the Z-pins are exposed to increased axial loading while the other $50 \%$ have increased bending and shear loading. For carbon fibre Z-pins, this configuration results in an increase in apparent fracture due to partial pull-out of the axially loaded specimens [10]. However, for the untreated stainless steel Z-pins tested in this study, increasing axial loading reduces snubbing effects which have been shown to increase energy absorption during pull-out failure. Therefore, arranging the stainless steel Z-pins in a $\pm \theta$ configuration resulted in a reduction in the apparent fracture toughness compared to orthogonally inserted pins. Nonetheless, on average, the energy absorption of inclined stainless steel Z-pin is still higher than that of inclined carbon fibre Z-pins.

Previous studies on Mode II fracture toughness tests showed that there were no significant effects of Z-pin material properties in 3ENF tests under fatigue loading [6]. However, it is likely that the differences reflected in the static tests here may be visible in fatigue tests conducted at higher displacement amplitudes. Thus Z-pinned structures reinforced with metal Z-pins, which are better at resisting large sliding displacements are likely to have superior fatigue properties as a system, compared to composite Zpinned structures whose small strains to failure have been shown to result in rupture at low fatigue cycle numbers [19]. 


\section{Conclusions}

In this study, the fracture toughness of inclined metal Z-pins has been analysed using single pin, DCB and ELS specimens. Under Mode I loads, inclined stainless steel Z-pins in a $\pm \theta$ degree configuration exhibit high energy absorption at low and high crack lengths due to a combination of enhanced friction due to snubbing and plastic deformation of the pins. Even at the low areal density analysed in this study, significantly high loads are required for Mode I delaminations to propagate through a Zpinned region reinforced with inclined metal Z-pins. Therefore, inclined stainless steel Z-pins are extremely effective TTR pins for Mode I loads, far better than any alternatives so far identified in the literature.

Inclined stainless steel Z-pins arranged a $\pm \theta$ configuration and tested under Mode II ELS loads have been shown to have a reduced apparent fracture toughness when compared to orthogonally inserted pins. This is primarily because for stainless steel Zpins without surface treatment, an increase in axial force, leads to failure under less effective energy absorption mechanisms. This finding differs from the case of inclined carbon fibre Z-pins where increasing axial forces leads to higher apparent fracture toughness. Therefore, this study has further highlighted the findings in previous studies [1]-[3][6] that the energy absorption of metallic Z-pins, due to a combination of plastic deformation and frictional pull-out, is a more effective approach to maximise bridging tractions under mixed Mode I/II loading than the traditional high strength, but more brittle, carbon fibre Z-pins. 


\section{Acknowledgements}

The authors would like to acknowledge Rolls-Royce plc and the Engineering and Physical Sciences Research Council (EPSRC) for their support of this research through the Composites University Technology Centre at the University of Bristol (UK) and Grant No.EP/G036772/1 respectively. Data from this study is not available, due to commercial sensitivity.

\section{References}

[1] H. Ji, J.-H. Kweon, and J.-H. Choi, "Fatigue characteristics of stainless steel pinreinforced composite hat joints," Compos. Struct., vol. 108, pp. 49-56, Feb. 2014.

[2] M.-G. Ko, J.-H. Kweon, and J.-H. Choi, "Fatigue characteristics of jagged pinreinforced composite single-lap joints in hygrothermal environments," Compos. Struct., vol. 119, pp. 59-66, Jan. 2015.

[3] K. Pingkarawat and A. P. Mouritz, "Comparative study of metal and composite z-pins for delamination fracture and fatigue strengthening of composites," Eng. Fract. Mech., Jan. 2016.

[4] D. D. R. Cartié, B. N. Cox, and N. A. Fleck, "Mechanisms of crack bridging by composite and metallic rods," Compos. Part A Appl. Sci. Manuf., vol. 35, no. 11, pp. 1325-1336, Nov. 2004.

[5] L. Tong and X. Sun, "Bending effect of through-thickness reinforcement rods on mode I delamination toughness of DCB specimen. I. Linearly elastic and rigidperfectly plastic models," Int. J. Solids Struct., vol. 41, no. 24-25, pp. 6831- 
6852, Dec. 2004.

[6] F. Pegorin, K. Pingkarawat, S. Daynes, and A. P. Mouritz, "Delamination Fatigue Properties of Z-Pinned Carbon-Epoxy Laminate Using Metal or Composite Rods," in 20th International Conference on Composite Materials (ICCM20), 2015.

[7] B. Zhang, G. Allegri, M. Yasaee, S. R. Hallett, and I. K. Partridge, "On the delamination self-sensing function of Z-pinned composite laminates," Compos. Sci. Technol., vol. 128, pp. 138-146, May 2016.

[8] B. Gu, H. Zhang, B. Wang, S. Zhang, and X. Feng, "Fracture toughness of laminates reinforced by piezoelectric z-pins," Theor. Appl. Fract. Mech., Jan. 2015.

[9] E. Greenhalgh and M. Hiley, "The assessment of novel materials and processes for the impact tolerant design of stiffened composite aerospace structures," Compos. Part A Appl. Sci. Manuf., vol. 34, no. 2, pp. 151-161, Feb. 2003.

[10] B. M'membe, S. Gannon, M. Yasaee, S. R. Hallett, and I. K. Partridge, "Mode II delamination resistance of composites reinforced with inclined Z-pins," Mater. Des., Jan. 2016.

[11] K. L. Rugg, B. N. Cox, K. E. Ward, and G. O. Sherrick, "Damage mechanisms for angled through-thickness rod reinforcement in carbon-epoxy laminates," Compos. Part A Appl. Sci. Manuf., vol. 29, no. 12, pp. 1603-1613, Dec. 1998.

[12] V. C. Li, Y. Wang, and S. Backer, "Effect of inclining angle, bundling and surface treatment on synthetic fibre pull-out from a cement matrix," Composites, vol. 21, no. 2, pp. 132-140, Mar. 1990. 
[13] J. Morton and G. W. Groves, "The cracking of composites consisting of discontinuous ductile fibres in a brittle matrix ? effect of fibre orientation," $J$. Mater. Sci., vol. 9, no. 9, pp. 1436-1445, Sep. 1974.

[14] M. Yasaee, J. K. Lander, G. Allegri, and S. R. Hallett, "Experimental characterisation of mixed mode traction-displacement relationships for a single carbon composite Z-pin,” Compos. Sci. Technol., Feb. 2014.

[15] ASTM D5528-13, "Standard Test Method for Mode I Interlaminar Fracture Toughness of Unidirectional Fiber-Reinforced Polymer Matrix Composites," ASTM International, West Conshohocken, 2013.

[16] S. Hashemi, A. J. Kinloch, and J. G. Williams, "The Analysis of Interlaminar Fracture in Uniaxial Fibre-Polymer Composites," Proc. R. Soc. A Math. Phys. Eng. Sci., vol. 427, no. 1872, pp. 173-199, Jan. 1990.

[17] ESIS-TC4, "Fibre-reinforced plastic composites - determination of apparent Mode II interlaminar fracture toughness, GIIc, for unidirectionally reinforced materials," ESIS-TC4 Eur. Struct. Integr. Soc. Comm., vol. 1-4-2, 2002.

[18] Y. Wang and J. Williams, "Corrections for mode II fracture toughness specimens of composites materials," Compos. Sci. Technol., vol. 43, no. 3, pp. 251-256, 1992.

[19] F. Warzok, G. Allegri, M. Gude, and S. R. Hallett, "Experimental characterisation of fatigue damage in single Z-pins," Compos. Part A Appl. Sci. Manuf., Mar. 2016.

[20] B. M'membe, M. Yasaee, S. R. Hallett, and I. K. Partridge, "Effect of Inclined Insertion on the Delamination Resistance of Z-Pinned Composites," in 16th 
European Conference on Composite Materials, 2014, no. June.

[21] K. P. Plain and L. Tong, "The effect of stitch incline angle on mode I fracture toughness - Experimental and modelling," Compos. Struct., vol. 92, no. 7, pp. 1620-1630, Jun. 2010.

[22] J. W. G. Treiber, "Performance of tufted carbon fibre/epoxy composites (PhD Thesis)," Cranfield University, 2011. 
2019-02-28

\section{Effective use of metallic Z-pins for composites' through-thickness reinforcement}

M'membe, Beene

Elsevier

M'membe B, Yasaee M, Hallett SR, Partridge IK. (2019) Effective use of metallic Z-pins for composites' through-thickness reinforcement. Composites Science and Technology, Volume 175, May 2019, pp. 77-84

http://doi.org/10.1016/j.compscitech.2019.02.024

Downloaded from Cranfield Library Services E-Repository 